TRANSACTIONS OF THE

AMERICAN MATHEMATICAL SOCIETY

Volume 351, Number 6, Pages 2183-2196

S 0002-9947(99)02387-9

Article electronically published on February 15, 1999

\title{
COMPACT COMPOSITION OPERATORS ON BMOA
}

\author{
P. S. BOURDON, J. A. CIMA, AND A. L. MATHESON
}

\begin{abstract}
We characterize the compact composition operators on BMOA, the space consisting of those holomorphic functions on the open unit disk $U$ that are Poisson integrals of functions on $\partial U$, that have bounded mean oscillation. We then use our characterization to show that compactness of a composition operator on BMOA implies its compactness on the Hardy spaces (a simple example shows the converse does not hold). We also explore how compactness of the composition operator $C_{\phi}:$ BMOA $\rightarrow$ BMOA relates to the shape of $\phi(U)$ near $\partial U$, introducing the notion of mean order of contact. Finally, we discuss the relationships among compactness conditions for composition operators on BMOA, VMOA, and the big and little Bloch spaces.
\end{abstract}

\section{INTRODUCTION}

Throughout this paper, $\phi$ will denote a holomorphic function taking the open unit disk $U$ into itself and $C_{\phi}$ will denote the operator of composition with $\phi$. Clearly, $C_{\phi}$ preserves the set $H(U)$ of holomorphic functions on $U$; that is,

$$
C_{\phi} f=f \circ \phi
$$

belongs to $H(U)$ whenever $f$ does. It is well known that $C_{\phi}$ also preserves many subspaces of $H(U)$ such as the classical Hardy spaces $H^{p}(U)$ and Bergman spaces $L_{a}^{p}(U)$. That $C_{\phi}$ also preserves BMOA is easy to verify (see [13] and, for example, the following section). Recall that the space BMOA consists of those holomorphic functions on the open unit disk $U$ that are Poisson integrals of functions on $\partial U$ that have bounded mean oscillation; also recall that BMOA $\subset H^{p}$ for every positive $p$.

The compactness problem for Hardy-space composition operators was solved in 1988 by J. H. Shapiro [9, Theorem 2.3], who characterized the compactness of $C_{\phi}: H^{p} \rightarrow H^{p}$ in terms of the Nevanlinna counting function of $\phi$ (Carleson measure considerations played a background role). In this paper, we characterize compact composition operators on BMOA via a Carleson measure condition. We then illustrate the utility of our characterization by employing it

- to show that compactness of $C_{\phi}:$ BMOA $\rightarrow$ BMOA implies compactness of $C_{\phi}$ on the Hardy spaces $H^{p}$, and

- to obtain a sharp sufficient condition for compactness of $C_{\phi}:$ BMOA $\rightarrow$ BMOA in terms of how $\phi(U)$ contacts $\partial U$.

Our "order of contact" condition shows that, for example, if $\phi(U)$ approaches $\partial U$ only within, say, the cusp bounded by the graph of $y=(x-1)^{2}$ and its reflection in

Received by the editors January 3, 1997 and, in revised form, March 17, 1998.

1991 Mathematics Subject Classification. Primary 47B38; Secondary 30D50.

The first author was supported in part by NSF grant DMS-9401206.

The third author was supported in part by NSF grant DMS-9500835. 
the $x$-axis, then $C_{\phi}$ will be compact on BMOA (similar conditions show that $C_{\phi}$ is compact on the Bloch space [7]). Our work on order of contact allows us to answer a question raised by Madigan and Matheson in [7] (see section 5 below).

In the final section of this paper we discuss how compactness of $C_{\phi}$ on BMOA relates to its compactness on the Bloch space and the little Bloch space. In particular, we comment on recent work of M. Tjani [14]. Tjani has shown that compactness of $C_{\phi}$ on BMOA implies its compactness on the Bloch space. In addition, she has obtained a sufficient condition for compactness of $C_{\phi}$ on BMOA involving the "counting function" for BMOA [14, Corollary 2.13] and has obtained a characterization of compact compositions operators on VMOA [14, Theorem 3.11] that is similar to one we present in section 3 of this paper.

\section{Preliminaries}

For a complex-valued function $f \in L^{1}(\partial U)$ and an $\operatorname{arc} I \in \partial U$, we let $f_{I}$ denote the average of $f$ over $I$, so that

$$
f_{I}=\frac{1}{|I|} \int_{I} f(\zeta) d m(\zeta)
$$

where $m$ denotes Lebesgue measure and $|I|=m(I)$ denotes the length of $I$. The space BMO consists of those functions $f \in L^{1}(\partial U)$ such that

$$
\|f\|_{b}:=\sup \left\{\frac{1}{|I|} \int_{I}\left|f(\zeta)-f_{I}\right| \operatorname{dm}(\zeta): I \text { is an } \operatorname{arc} \text { of } \partial U\right\}
$$

is finite. For $f \in L^{1}(\partial U)$, we will freely identify $f$ with its Poisson extension into $U$ so that if $a \in U, f(a)=P[f](a)$, where $P$ denotes the Poisson kernel for $U$. The space BMO is a Banach space with norm

$$
f \rightarrow\|f\|_{b}+|f(0)|
$$

Clearly, $L^{\infty}(\partial U) \subset$ BMO; in fact, harmonic conjugates of functions in $L^{\infty}(\partial U)$ belong to BMO and every function $f$ in $\mathrm{BMO}$ has the representation

$$
f=f_{1}+\tilde{f}_{2}
$$

where $f_{1}$ and $f_{2}$ are in $L^{\infty}(\partial U)$ and $\tilde{f}_{2}$ denotes the harmonic conjugate of $f_{2}$ that vanishes at 0 (see, for example, [8, p. 39]).

The representation (2.2) shows that BMO is preserved by composition with $\phi$ for any holomorphic self-map of the unit disk: Clearly $f_{1} \circ \phi$ remains bounded; moreover, $\tilde{f}_{2} \circ \phi=\widetilde{f_{2} \circ \phi}+\tilde{f}_{2}(\phi(0))$. The closed graph theorem now shows that $C_{\phi}: \mathrm{BMO} \rightarrow \mathrm{BMO}$ is bounded.

In this paper, our primary focus will be on composition operators acting on the closed subspace BMOA of BMO. The space BMOA consists of those holomorphic functions on $U$ that are Poisson integrals of BMO functions. That $C_{\phi}$ is a bounded linear operator on BMOA follows immediately from the discussion of the preceding paragraph (because composition with $\phi$ preserves analyticity). We now obtain a bound on the operator norm of $C_{\phi},\left\|C_{\phi}\right\|$. The BMO norm defined by (2.1) is equivalent to the norm $\|\cdot\|_{s}$ defined by

$$
\|f\|_{s}=\inf \left\{\max \left\{\left\|f_{1}\right\|_{\infty},\left\|f_{2}\right\|_{\infty}\right\}: f=f_{1}+\tilde{f}_{2}, f_{1} \text { and } f_{2} \in L^{\infty}\right\}
$$


(see, e.g., [8, p. 46]); also, there is a constant $C>1$ such that if $g \in L^{\infty}(\partial U)$ and $z \in U$,

$$
|\tilde{g}(z)| \leq C\|g\|_{\infty} \log \left(\frac{1}{1-|z|}\right) .
$$

Thus, if $f=f_{1}+\tilde{f}_{2}\left(f_{1}\right.$ and $f_{2}$ in $\left.L^{\infty}(\partial U)\right)$ is a BMOA (or BMO) function, then $f \circ \phi=f_{1} \circ \phi+\tilde{f}_{2}(\phi(0))+\widetilde{f_{2} \circ \phi}$; and using the norm representation (2.3), we have

$$
\begin{aligned}
\|f \circ \phi\|_{s} & \leq \max \left\{\left\|f_{1} \circ \phi+\tilde{f}_{2}(\phi(0))\right\|_{\infty},\left\|f_{2} \circ \phi\right\|_{\infty}\right\} \\
& \leq \max \left\{\left\|f_{1}\right\|_{\infty},\left\|f_{2}\right\|_{\infty}\right\}+C\left\|f_{2}\right\|_{\infty} \log \left(\frac{1}{1-|\phi(0)|}\right) \\
& \leq C \max \left\{\left\|f_{1}\right\|_{\infty},\left\|f_{2}\right\|_{\infty}\right\}\left[1+\log \left(\frac{1}{1-|\phi(0)|}\right)\right] .
\end{aligned}
$$

Because $f_{1}+\tilde{f}_{2}$ is an arbitrary representation of $f$ as an $L^{\infty}$ function plus a conjugate $L^{\infty}$ function, it follows that

$$
\left\|C_{\phi}\right\| \leq C\left[1+\log \left(\frac{1}{1-|\phi(0)|}\right)\right] .
$$

This bound on the operator norm of $C_{\phi}$ should be compared with the bound for $C_{\phi}: H^{p} \rightarrow H^{p}$ for $p \geq 1$ (see, e.g. [3, p. 125]):

$$
\left\|C_{\phi}\right\|_{H^{p}} \leq\left(\frac{1+|\phi(0)|}{1-|\phi(0)|}\right)^{1 / p} .
$$

Our characterization of compact composition operators on BMOA (and BMO) involves Carleson measures. For an arc $I$ of the unit circle, we let $R(I)$ denote the Carleson rectangle determined by $I$ so that

$$
R(I)=\left\{r e^{i \theta}: 1-|I| / 2 \pi<r<1 \text { and } e^{i \theta} \in I\right\} .
$$

A finite positive measure $\mu$ on $U$ is a Carleson measure provided that

$$
\nu(\mu):=\sup \left\{\frac{\mu(R(I))}{|I|}: I \text { is a subarc of } \partial U\right\}
$$

is finite, in which case we call $\nu(\mu)$ the Carleson constant of $\mu$.

Carleson measures $\mu$ are those measures for which the Poisson integral gives a bounded map from $L^{p}(\partial U, d m)$ to $L^{p}(U, d \mu), 1<p<\infty$. This is the content of Carleson's Embedding Theorem (see, e.g., [8, p. 5]). We use the following consequence of Carleson's theorem in our proof that compactness of $C_{\phi}$ on BMOA implies its compactness on $H^{2}$ (hence on all of the Hardy spaces [11, §6]).

Theorem 2.1 (Carleson). There is a constant $C$ such that for each $f \in H^{2}$ and every Carleson measure $\mu$,

$$
\int_{U}|f|^{2} d \mu \leq C \nu(\mu)\|f\|_{H^{2}}^{2} .
$$

Carleson measures may be used to characterize functions in BMO. For $f \in$ $L^{1}(\partial U)$, let $\mu_{f}$ be defined by

$$
d \mu_{f}(z)=(1 / 2)|(\nabla f)(z)|^{2}(1-|z|) d A(z),
$$

where $d A$ denotes Lebesgue area measure. 
Theorem 2.2 (Fefferman). For a function $f \in L^{1}(\partial U)$, the following are equivalent:

(a) $f \in \mathrm{BMO}$;

(b) The measure $\mu_{f}$ is a Carleson measure.

Thus $f \in$ BMOA if and only if

$$
d \mu_{f}(z)=\left|f^{\prime}(z)\right|^{2}(1-|z|) d A(z)
$$

is a Carleson measure. The proof of Fefferman's theorem (see, e.g, [8, Chapter 4]) shows that the norm $\|\cdot\|_{*}$ on BMO defined by

$$
\|f\|_{*}=\left(\nu\left(\mu_{f}\right)\right)^{1 / 2}+|f(0)| \text {. }
$$

is equivalent to the two norms for BMO discussed above.

A function $f \in \mathrm{BMO}$ is said to have vanishing mean oscillation and belong to VMO provided

$$
\lim _{|I| \rightarrow 0} \frac{\mu_{f}(R(I))}{|I|}=0
$$

The space VMO is not always preserved under composition with an analytic selfmap $\phi$ of $U$. Because $f(z)=z$ belongs to VMO, an obvious necessary condition for preservation under $C_{\phi}$ is that $\phi$ belong to VMO. Since VMO $\cap L^{\infty}$ is an algebra [6, p. 377], it's not difficult to see that, in fact, $\phi \in \mathrm{VMO}$ is sufficient to ensure $C_{\phi}$ map VMO into itself.

Proposition 2.3. The operator $C_{\phi}$ maps $\mathrm{VMO}$ into itself if and only if $\phi \in \mathrm{VMO}$.

We let VMOA denote the collection of those functions in VMO that are analytic on $U$. The preceding proposition (together with the closed graph theorem) shows $C_{\phi}$ is a bounded linear operator on VMOA. Observe that any univalent (holomorphic) function $f$ mapping $U$ onto a region of finite area will satisfy condition (2.4) and thus belong to VMOA. Hence, whenever the self-map $\phi$ of $U$ is univalent, $C_{\phi}$ will preserve VMOA.

\section{COMPaCtNess CHARACTERIZATION}

In this section we characterize the compact composition operators on BMO. Not surprisingly, compactness on BMO is equivalent to compactness on BMOA. We present our arguments in the BMOA setting and then discuss modifications needed for the BMO setting. In what follows $\Omega_{r}$ represents $\{z \in U:|\phi(z)|>r\}$ while $\tilde{\Omega}_{r}=U \backslash \Omega_{r}$. The characteristic function of $\Omega_{r}$ will be denoted by $1_{\Omega_{r}}$.

Theorem 3.1. The composition operator $C_{\phi}$ is compact on BMOA if and only if for every $\epsilon>0$ there is an $r, 0<r<1$, such that

$$
\int_{R(I)} 1_{\Omega_{r}}(z)\left(1-|z|^{2}\right)\left|f^{\prime}(\phi(z))\right|^{2}\left|\phi^{\prime}(z)\right|^{2} d A(z) \leq \epsilon|I|
$$

for every arc $I$ and every $f \in$ BMOA with $\|f\|_{*} \leq 1$.

Proof of sufficiency. Let $\left(f_{n}\right)$ be a sequence in the unit ball of BMOA which converges to 0 uniformly on compact subsets of $U$. It will be enough to show that $f_{n} \circ \phi$ converges to 0 in norm. Fix $\epsilon>0$ and let $r, 0<r<1$, be such that (3.1) holds. Since $\phi\left(\tilde{\Omega}_{r}\right)$ is a relatively compact subset of $U, f_{n} \circ \phi$ and $f_{n}^{\prime} \circ \phi$ converge 
uniformly to 0 on $\tilde{\Omega}_{r}$. In particular there is an $N$ such that $\left|f_{n}^{\prime} \circ \phi(z)\right|^{2}<\epsilon$ if $n \geq N$ and $z \in \tilde{\Omega}_{r}$. Consequently

$$
\int_{R(I)} 1_{\tilde{\Omega}_{r}}(z)\left(1-|z|^{2}\right)\left|f_{n}^{\prime}(\phi(z))\right|^{2}\left|\phi^{\prime}(z)\right|^{2} d A(z) \leq \epsilon\|\phi\|_{*}^{2}|I|
$$

for all $n \geq N$. On the other hand,

$$
\int_{R(I)} 1_{\Omega_{r}}(z)\left(1-|z|^{2}\right)\left|f_{n}^{\prime}(\phi(z))\right|^{2}\left|\phi^{\prime}(z)\right|^{2} d A(z) \leq \epsilon|I|
$$

for all $n$ by our choice of $r$. Hence, for $n \geq N$,

$$
\int_{R(I)}\left(1-|z|^{2}\right)\left|f_{n}^{\prime}(\phi(z))\right|^{2}\left|\phi^{\prime}(z)\right|^{2} d A(z) \leq\left(\|\phi\|_{*}^{2}+1\right) \epsilon|I|,
$$

and since $f_{n} \circ \phi(0) \rightarrow 0$, it follows that $\left\|f_{n} \circ \phi\right\|_{*} \rightarrow 0$. Thus $C_{\phi}$ is compact.

The proof that condition (3.1) is necessary for compactness of $C_{\phi}:$ BMOA $\rightarrow$ BMOA will depend on the following two lemmas.

Lemma 3.2. Suppose $C_{\phi}$ is compact on BMOA. Then for every $\epsilon>0$ there is an $r, 0<r<1$, such that

$$
\int_{R(I)} 1_{\Omega_{r}}(z)\left(1-|z|^{2}\right)\left|\phi^{\prime}(z)\right|^{2} d A(z)<\epsilon|I|
$$

for all arcs $I$ on $\partial U$.

Proof. Since $z^{n}$ is norm bounded in BMOA and converges uniformly to 0 on compact subsets of $U$, and since $C_{\phi}$ is compact, $\left\|\phi^{n}\right\|_{*} \rightarrow 0$. Hence, given $\epsilon>0$, there is an $N>1$ such that if $n \geq N$, then

$$
n^{2} \int_{R(I)}|\phi(z)|^{2 n-2}\left(1-|z|^{2}\right)\left|\phi^{\prime}(z)\right|^{2} d A(z)<\epsilon|I|
$$

for all $I$. Given $r, 0<r<1$,

$$
\begin{aligned}
& N^{2} r^{2 N-2} \int_{R(I)} 1_{\Omega_{r}}(z)\left(1-|z|^{2}\right)\left|\phi^{\prime}(z)\right|^{2} d A(z) \\
& \quad \leq N^{2} \int_{R(I)}|\phi(z)|^{2 N-2}\left(1-|z|^{2}\right)\left|\phi^{\prime}(z)\right|^{2} d A(z)<\epsilon|I|
\end{aligned}
$$

for any $I$, since $|\phi(z)|>r$ on $\Omega_{r}$. Choosing $r$ so that $N^{2} r^{2 N-2}=1$ proves the lemma.

Lemma 3.3. Suppose that $C_{\phi}$ is compact on BMOA and that $f \in \mathrm{BMOA}$. Then for every $\epsilon>0$ there exists $r=r(f, \epsilon), 0<r<1$, such that

$$
\int_{R(I)} 1_{\Omega_{r}}(z)\left(1-|z|^{2}\right)\left|f^{\prime}(\phi(z))\right|^{2}\left|\phi^{\prime}(z)\right|^{2} d A(z)<\epsilon|I|
$$

for every arc $I$.

Proof. Let $f_{s}(z)=f(s z)$ for $0<s<1$. Then $f_{s} \rightarrow f$ uniformly on compact subsets of $U$ as $s \rightarrow 1$ and the family $\left\{f_{s}: 0<s<1\right\}$ is bounded in BMOA; thus, $\left\|f_{s} \circ \phi-f \circ \phi\right\|_{*} \rightarrow 0$. In particular, for each $\epsilon>0$ there is an $s, 0<s<1$, such that

$$
\int_{R(I)}\left(1-|z|^{2}\right)\left|f_{s}^{\prime}(\phi(z))-f^{\prime}(\phi(z))\right|^{2}\left|\phi^{\prime}(z)\right|^{2} d A(z)<\frac{\epsilon}{4}|I| .
$$


On the other hand,

$$
\begin{aligned}
& \int_{R(I)} 1_{\Omega_{r}}(z)\left(1-|z|^{2}\right)\left|f_{s}^{\prime}(\phi(z))\right|^{2}\left|\phi^{\prime}(z)\right|^{2} d A(z) \\
& \quad \leq\left\|f_{s}^{\prime}\right\|_{\infty}^{2} \int_{R(I)} 1_{\Omega_{r}}(z)\left(1-|z|^{2}\right)\left|\phi^{\prime}(z)\right|^{2} d A(z)<\frac{\epsilon}{4}|I|
\end{aligned}
$$

for an appropriate choice of $r, 0<r<1$, by Lemma 3.2. Lemma 3.3 follows on applying the triangle inequality.

Proof of necessity (Theorem 3.1). Assume that $C_{\phi}$ is compact on BMOA. Let $B$ denote the unit ball of BMOA. Since $C_{\phi}(B)$ is relatively compact in BMOA, there is, for each $\epsilon>0$, a finite set of functions $f_{1}, \ldots, f_{n}$ in $B$ such that for each $f \in B$ there is an $i, i=1, \ldots, n$, such that

$$
\int_{R(I)}\left(1-|z|^{2}\right)\left|f^{\prime}(\phi(z))-f_{i}^{\prime}(\phi(z))\right|^{2}\left|\phi^{\prime}(z)\right|^{2} d A(z)<\frac{\epsilon}{4}|I|
$$

for each $\operatorname{arc} I$. Applying Lemma 3.3 to the functions $f_{1}, \ldots, f_{n}$ yields $r=$ $\max _{1 \leq i \leq n} r\left(f_{i}, \epsilon / 4\right)$ such that

$$
\int_{R(I)} 1_{\Omega_{r}}(z)\left(1-|z|^{2}\right)\left|f_{i}^{\prime}(\phi(z))\right|^{2}\left|\phi^{\prime}(z)\right|^{2} d A(z)<\frac{\epsilon}{4}|I|
$$

for each $\operatorname{arc} I$ and for $i=1, \ldots, n$. The triangle inequality completes the proof.

The decomposition of BMO functions provided by Fefferman's theorem into sums of $L^{\infty}$ and conjugate $L^{\infty}$ functions leads to the following improvement of Theorem 3.1 .

Theorem 3.4. Let $\phi$ be a holomorphic mapping of the unit disk into itself, with corresponding composition operator $C_{\phi}$. Then the following are equivalent:

(a) $C_{\phi}$ is compact on $\mathrm{BMO}$;

(b) $C_{\phi}$ is compact on BMOA;

(c) for every $\epsilon>0$ there is an $r, 0<r<1$, such that

$$
\int_{R(I)} 1_{\Omega_{r}}(z)\left(1-|z|^{2}\right)|\nabla f(\phi(z))|^{2}\left|\phi^{\prime}(z)\right|^{2} d A(z) \leq \epsilon|I|
$$

for all arcs $I$ on $\partial U$ and for every $f \in L^{\infty}$ with $\|f\|_{\infty} \leq 1$.

Proof. That (a) implies (b) is clear. To see that (b) implies (a) observe that if $f \in \mathrm{BMO}$, then $f=\Re g+i \Re h$ for BMOA functions $g$ and $h$ with $g(0)=\Re f(0)$ and $h(0)=\Im f(0)$. Observe that because $|\nabla \Re g|=\left|g^{\prime}\right|$,

$$
\begin{aligned}
\|g\|_{*} & =|g(0)|+\left(\sup _{I}\left\{\frac{1}{|I|} \int_{R(I)}\left|g^{\prime}(z)\right|^{2}(1-|z|) d A(z)\right\}\right)^{1 / 2} \\
& \leq|\Re f(0)|+\left(2 \sup _{I}\left\{\frac{1}{|I|} \int_{R(I)}(1 / 2)|\nabla \Re g|^{2}(1-|z|) d A(z)\right\}\right)^{1 / 2} \\
& \leq 2\|f\|_{*} .
\end{aligned}
$$

The same argument shows that $\|h\|_{*} \leq 2\|f\|_{*}$. Thus a norm bounded sequence $\left(f_{n}\right)$ in BMO corresponds to a pair of norm bounded sequences $\left(g_{n}\right)$ and $\left(h_{n}\right)$ in BMOA with $f_{n}=\Re g_{n}+i \Re h_{n}$. If $C_{\phi}$ is compact on BMOA, we can find a subsequence $\left(r_{n}\right)$ 
of the sequence of positive integers such that both $\left(g_{r_{n}} \circ \phi\right)$ and $\left(h_{r_{n}} \circ \phi\right)$ converge in norm, and thus $\left(f_{r_{n}} \circ \phi\right)$ will converge in norm as well. Hence (b) implies (a).

That (b) implies (c) follows quickly from Theorem 3.1 and the decomposition of BMO functions discussed in the preceding paragraph. Because $L^{\infty}(\partial U) \subset$ BMO, there is a constant $C_{\infty}$ such that $\|f\|_{*} \leq C_{\infty}\|f\|_{\infty}$ for each $f \in L^{\infty}$. Let $f$ be an arbitrary element of the unit ball of $L^{\infty}(\partial U)$ and as in the preceding paragraph write $f=\Re g+i \Re h$, where $g$ and $h$ are BMOA functions such that $\|g\|_{*} \leq 2\|f\|_{*}$ and $\|h\|_{*} \leq 2\|f\|_{*}$. Because $\|f\|_{\infty} \leq 1$, we have that both $\|g\|_{*}$ and $\|h\|_{*}$ do not exceed $2 C_{\infty}$. Theorem 3.1 now shows (b) implies (c).

That (c) implies (b) remains to be proved. Because the norms $\|\cdot\|_{s}$ and $\|\cdot\|_{*}$ are equivalent, there is a constant $C$ such that $\|f\|_{s} \leq C\|f\|_{*}$ for each $f \in$ BMOA. Given $\epsilon>0$, choose $r$ so that the integral on the left of (3.2) is less than $\epsilon|I| /\left(16 C^{2}\right)$. Suppose $f$ is a function in BMOA with $\|f\|_{*}=1$. Let $f_{1}$ and $f_{2}$ be $L^{\infty}$ functions such that $f=f_{1}+\tilde{f}_{2}$ and $\max \left\{\left\|f_{1}\right\|_{\infty},\left\|f_{2}\right\|_{\infty}\right\} \leq(2 C)\|f\|_{*}$. Because $\left|\nabla \tilde{f}_{2}\right|=\left|\nabla f_{2}\right|$, one can easily verify the condition of Theorem 3.1 holds so that $C_{\phi}$ is compact on BMOA.

The compactness condition of the preceding theorem takes a simpler form in the VMO setting. Because every $f \in \mathrm{VMO}$ has a decomposition $f=g+\tilde{h}$, where $g$ and $h$ are continuous on $\partial U$ (see, e.g., [8, p. 50]), in condition (c), one may replace the collection of bounded functions on the circle with the collection $C(\partial U)$ of continuous functions on the circle. Here is the statement of the analogue of Theorem 3.4 for the VMO setting; we omit the proof.

Theorem 3.5. Let $\phi \in \mathrm{VMOA}$ be a holomorphic mapping of the unit disk into itself. Then the following are equivalent:

(a) $C_{\phi}$ is compact on VMO;

(b) $C_{\phi}$ is compact on VMOA;

(c) for every $\epsilon>0$ there is a $\delta>0$ such that

$$
\int_{R(I)}\left(1-|z|^{2}\right)|\nabla f(\phi(z))|^{2}\left|\phi^{\prime}(z)\right|^{2} d A(z) \leq \epsilon|I|
$$

whenever $I$ is an arc on $\partial U$ with $|I| \leq \delta$ and $f \in C(\partial U)$ with $\|f\|_{\infty} \leq 1$.

\section{Compactness on BMOA versus compactness on $H^{2}$}

In this section we use our BMOA compactness characterization of Theorem 3.1 to prove that if $C_{\phi}$ is compact on BMOA, then it is also compact on the Hardy spaces $H^{p}(U)$. We conclude the section with an example showing that compactness of $C_{\phi}$ on the Hardy spaces does not imply its compactness on BMOA.

Compact composition operators in the Hardy space setting were characterized by Shapiro in [9]; using his work, Cima and Matheson point out in [2] that $C_{\phi}$ is compact on $H^{2}$ if and only if

$$
\left\|C_{\phi} k_{a}\right\|_{H^{2}} \rightarrow 0 \quad \text { as } \quad|a| \rightarrow 1^{-},
$$

where

$$
k_{a}=\frac{\sqrt{1-|a|^{2}}}{1-\bar{a} z}
$$

is the normalized reproducing kernel at $a \in U$ for $H^{2}$. 
We show that if $C_{\phi}$ is compact on BMOA, then condition (4.1) holds. Our proof employs Carleson's Embedding Theorem (stated as Theorem 2.1 in section 2). We also make use of the following well-known fact (which follows quickly from the Littlewood-Paley identity ([6, Chapter 6$])$ or may be established directly using power series): For $f \in H^{2}$,

$$
\begin{aligned}
& 8\left(|f(0)|^{2}+\int_{U}\left|f^{\prime}(z)\right|^{2}\left(1-|z|^{2}\right) d A(z) / \pi\right) \\
& \quad \geq\|f\|_{H^{2}}^{2} \geq|f(0)|^{2}+\int_{U}\left|f^{\prime}(z)\right|^{2}\left(1-|z|^{2}\right) d A(z) / \pi .
\end{aligned}
$$

Theorem 4.1. Suppose $C_{\phi}: \mathrm{BMOA} \rightarrow \mathrm{BMOA}$ is compact. Then $C_{\phi}: H^{2} \rightarrow H^{2}$ is compact.

Proof. Let $M$ denote the operator norm of $C_{\phi}: H^{2} \rightarrow H^{2}$. For $a \in U$, let $L_{a}(z)=$ $\log (1-\bar{a} z)$, and let

$$
d \mu_{r, a}(z)=1_{\Omega_{r}}(z)|a|^{2}\left|\phi^{\prime}(z)\right|^{2} \frac{1-|z|^{2}}{|1-\bar{a} \phi(z)|^{2}} d \lambda(z),
$$

where $d \lambda=d A / \pi, 0<r<1$, and, as before, $\Omega_{r}=\{z \in U:|\phi(z)|>r\}$. Let $\epsilon>0$. Because the family $\left\{L_{a}: a \in U\right\}$ is bounded in BMOA, Theorem 3.1 shows that there is an $r \in(0,1)$ such that for each $a \in U$,

$$
\nu\left(\mu_{r, a}\right)<\frac{\epsilon^{2}}{C M^{2}}
$$

here, $C$ is the constant of Theorem 2.1 .

Now choose $s \in(0,1)$ large enough so that when $1>t>s$, both $\sqrt{1-t^{2}} /$ $(1-t|\phi(0)|)=k_{t}(|\phi(0)|)<\epsilon$ and $\frac{1-t^{2}}{(1-t r)^{4}}<\epsilon^{2}$. As long as $a \in U$ satisfies $|a|>s$, we have

$$
\begin{aligned}
(1 / 8)\left\|C_{\phi} k_{a}\right\|_{H^{2}}^{2} \leq & \left|k_{a}(\phi(0))\right|^{2}+\int_{U} \frac{1-|a|^{2}}{|1-\bar{a} \phi(z)|^{4}}|a|^{2}\left|\phi^{\prime}(z)\right|^{2}\left(1-|z|^{2}\right) d \lambda(z) \\
\leq & \epsilon^{2}+\int_{\{|\phi| \leq r\}} \frac{1-|a|^{2}}{|1-\bar{a} \phi(z)|^{4}}\left|\phi^{\prime}(z)\right|^{2}\left(1-|z|^{2}\right) d \lambda(z) \\
& +\int_{U} \frac{1-|a|^{2}}{|1-\bar{a} \phi|^{2}} d \mu_{a, r}(z) \\
\leq & \epsilon^{2}+\epsilon^{2}\|\phi\|_{H^{2}}^{2}+\left\|k_{a}(\phi)\right\|_{L^{2}\left(\mu_{a, r}\right)}^{2} \\
\leq & 2 \epsilon^{2}+\left(\epsilon^{2} / M^{2}\right)\left\|k_{a}(\phi)\right\|_{H^{2}}^{2} \\
\leq & 3 \epsilon^{2}
\end{aligned}
$$

where, to obtain the next-to-last inequality, we have used Theorem 2.1 and $\|\phi\|_{H^{2}} \leq$ 1 (because $\phi$ is a self-map of $U$ ). It follows that $\left\|C_{\phi} k_{a}\right\|_{H^{2}} \rightarrow 0$ as $|a| \rightarrow 1^{-}$so that $C_{\phi}$ is compact on $H^{2}$.

The converse of the preceding theorem is not valid. Our argument depends upon the following proposition.

Proposition 4.2. If $\phi \in \mathrm{VMOA}$ and $C_{\phi}: \mathrm{BMOA} \rightarrow \mathrm{BMOA}$ is compact, then $C_{\phi}$ maps BMOA into VMOA. 
Even weak compactness of $C_{\phi}: \mathrm{VMOA} \rightarrow \mathrm{VMOA}$ is sufficient to ensure that $C_{\phi}$ map BMOA into VMOA. Recall that the bounded linear operator $T$ from the Banach space $X$ to the Banach space $Y$ is weakly compact provided that the weak closure of the image under $T$ of the unit ball in $X$ is a weakly compact set in $Y$. According to Gantmacher's theorem $T: X \rightarrow Y$ is weakly compact if and only if $T^{* *}\left(X^{* *}\right)$ is contained in the natural embedding of $Y$ into $Y^{* *}$ (see, e.g., [4, p. 482]). Because the second dual of VMOA is BMOA, it follows that for $\phi \in \mathrm{VMOA}, C_{\phi}: \mathrm{VMOA} \rightarrow \mathrm{VMOA}$ is weakly compact if and only if $f \circ \phi \in \mathrm{VMOA}$ for all $f \in$ BMOA.

The following example shows that compactness of $C_{\phi}$ on $H^{2}$ does not imply compactness of $C_{\phi}$ on BMOA. (Madigan and Matheson use essentially the same example and the same argument in [7] to show that, for instance, compactness of $C_{\phi}$ on $H^{2}$ does not imply its compactness on the Bloch space.)

Example 4.3. Let $\phi(z)=1-\sqrt{1-z}$ so that $\phi$ maps the disk $U$ univalently onto the region pictured in Figure 1. Because $\phi$ is univalent (or because it is continuous on $\partial U$ ), it is in VMOA. Observe that

$$
\log (1-\phi(z))=1 / 2 \log (1-z)
$$

and thus $C_{\phi}$ does not map BMOA into VMOA and cannot be compact on BMOA by Proposition 4.2. However, because $\phi$ is univalent and fails to have angular derivative at each point of $\partial U$, the operator $C_{\phi}: H^{2} \rightarrow H^{2}$ is compact (see, e.g., $[10$, p. 57])).

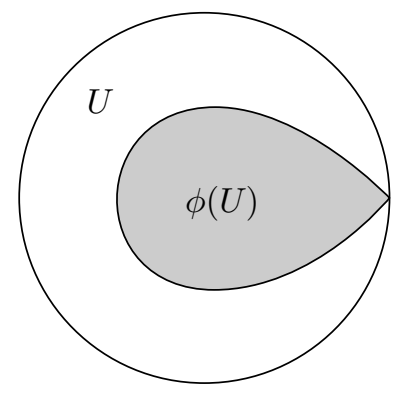

Figure 1. The image of $\phi(z)=1-(1-z)^{1 / 2}$.

\section{ORDER OF CONTACT AND COMPACTNESS}

For $\alpha>0$ and $G$ an open subset of $U$, we say that $G$ contacts $\partial U$ with mean order (at most) $\alpha$ provided that

$$
\int_{0}^{2 \pi} 1_{G}\left(r e^{i \theta}\right) d \theta=\mathrm{O}\left((1-r)^{1 / \alpha}\right)
$$

as $r \rightarrow 1^{-}$, where $1_{G}$ is the characteristic function of the set $G$. Observe that the integral on the left of (5.1) represents the angular measure of $G$ intersected with the circle $\{z:|z|=r\}$.

Our notion of mean order of contact is consistent with the usual pointwise orderof-contact concept (cf. [10, p. 45]). For example, the mean order of contact for a domain contained in a Stoltz region is at most 1. "Cuspy" contact corresponds to 
orders less than one. For instance, if a domain $G \subset U$ has boundary contacting the circle only at 1 and near $1, G$ lies between the graph of $y=(1-x)^{2}$ and its reflection in the $x$-axis, then $G$ makes contact with $\partial U$ with mean-order at most $1 / 2$.

We now make precise the connection between mean order of contact and pointwise order of contact. Let 1 be a point of $\partial G \cap \partial U$; we say that $G$ contacts $\partial U$ at 1 with order at most $b$ provided that

$$
\frac{|1-z|^{b}}{1-|z|}
$$

remains bounded as $z$ approaches 1 within $G$.

Let $G$ be a subregion of $U$ whose boundary touches the unit circle precisely at 1 with order of contact $b$ at 1 . Observe that we must have

$$
\sup \left\{|1-z|^{b} /(1-|z|): z \in G\right\} \leq C
$$

for some constant $C$. Because $G$ contacts the circle only at 1 , there is an $r_{0}$ such that all points in $G$ with modulus greater than $r_{0}$ lie in the right-half plane. Let $r>r_{0}$ and let $z=r e^{i t}$ be a point in $G$. Because $z$ is necessarily in the right half-plane, we may take $t \in(-\pi / 2, \pi / 2)$. We have

$$
|r t / 2|^{b} \leq|r \sin (t)|^{b} \leq\left|1-r e^{i t}\right|^{b} .
$$

The preceding inequality combined with (5.2) yields

$$
|t|<C^{1 / b}(2 / r)(1-r)^{1 / b} .
$$

Thus $G \cap\{z:|z|=r\}$ must lie within the sector $-C^{1 / b}(2 / r)(1-r)^{1 / b}<$ $\operatorname{Arg} z<C^{1 / b}(2 / r)(1-r)^{1 / b}$ so that the angular measure of $G$ intersect the circle of radius $r$ is bounded by a constant times $(1-r)^{1 / b}$. Thus for a domain whose boundary contacts the circle at exactly one point, mean order of contact corresponds to pointwise order of contact.

Shapiro and Taylor [11] appear to have been the first to point out an explicit connection between order of contact of $\phi(U)$ and compactness of the corresponding composition operator on $H^{2}$. They showed that if $\phi(U)$ is contained in a polygon inscribed in the unit circle, then $C_{\phi}$ will be compact on $H^{2}$. Example 4.3 of the preceding section shows that the containment of $\phi(U)$ in a polygon circumscribed by the unit circle is not sufficient to ensure that $C_{\phi}$ be compact on BMOA. Thus, contact of $\phi(U)$ with $\partial U$ of mean order 1 is not sufficient to guarantee that $C_{\phi}$ : $\mathrm{BMOA} \rightarrow \mathrm{BMOA}$ is compact. We will show, however, that mean-order of contact less than one does guarantee compactness in the BMOA setting. Our work depends on the following.

Proposition 5.1. Suppose that $\phi$ is a holomorphic mapping of $U$ into itself which satisfies

$$
\int_{U} \frac{\left|\phi^{\prime}(z)\right|^{2}}{\left(1-|\phi(z)|^{2}\right)^{2}} d A(z)<\infty
$$

then $C_{\phi}$ is compact on BMOA.

Proof. If $f \in \mathrm{BMOA}$, then

$$
\left|f^{\prime}(z)\right| \leq C \frac{\|f\|_{*}}{1-|z|^{2}}
$$


for all $z \in U$, where $C$ is an absolute constant (see, e.g. [8, p. 92]). Thus if $\|f\|_{*} \leq 1$, then

$$
\frac{1}{|I|} \int_{R(I)} 1_{\Omega_{r}}(z)\left|f^{\prime}(\phi(z))\right|^{2}\left|\phi^{\prime}(z)\right|^{2}(1-|z|) d A(z) \leq C \int_{U} 1_{\Omega_{r}}(z) \frac{\left|\phi^{\prime}(z)\right|^{2}}{\left(1-|\phi(z)|^{2}\right)^{2}} d A(z)
$$

because $(1-|z|) /|I|<1 /(2 \pi)$ for $z \in R(I)$ and the integral can only increase if we then replace $R(I)$ with the larger region of integration $U$. The integral on the right tends to zero as $r$ tends to 1 since the measure of $\Omega_{r}$ tends to zero and the integrand is integrable over $\mathrm{U}$. Thus the condition of Theorem 3.1 is satisfied and $C_{\phi}$ is compact on BMOA.

The condition (5.4) appears in Shapiro and Taylor's paper [11] as a necessary and sufficient condition for $C_{\phi}$ to be Hilbert-Schmidt on the classical Dirichlet space of $U$. M. Tjani recently showed ([14, Proposition 3.9]) that condition (5.4) implies $C_{\phi}$ is compact from the Bloch space to BMOA (from which Proposition 5.1 follows).

Corollary 5.2. Suppose $\phi$ is univalent and $\phi(U)$ makes contact with the unit circle with mean order $\alpha<1$. Then $C_{\phi}$ is compact on BMOA.

Proof. Let $G=\phi(U)$. Because $G$ makes contact with $\partial U$ with mean order $\alpha$ there is a constant $C$ such that

$$
\int_{0}^{2 \pi} 1_{G}\left(r e^{i \theta}\right) d \theta \leq C(1-r)^{1 / \alpha}
$$

for every $r, 0 \leq r<1$. Changing variables and using polar coordinates yields

$$
\begin{aligned}
\int_{U} \frac{\left|\phi^{\prime}(z)\right|^{2}}{\left(1-|\phi(z)|^{2}\right)^{2}} d A(z) & =\int_{G} \frac{1}{\left(1-|z|^{2}\right)^{2}} d A(z) \\
& =\int_{0}^{1} \int_{0}^{2 \pi} \frac{1_{G}\left(r e^{i \theta}\right)}{\left(1-r^{2}\right)^{2}} d \theta d r \\
& \leq C \int_{0}^{1}(1-r)^{\frac{1}{\alpha}-2} d r
\end{aligned}
$$

which is finite because $\alpha<1$. Thus, by Proposition 5.1, $C_{\phi}$ is compact.

Corollary 5.3. If $\phi(U)$ is contained in a simply connected region which contacts the unit circle with mean order $\alpha<1$, then $C_{\phi}$ is compact on BMOA.

Proof. Suppose that $\phi(U)$ is contained in the simply connected region $G \subset U$ and that $G$ contacts the unit circle with order $\alpha<1$. Let $\psi$ map $U$ univalently onto $G$. Then $C_{\psi}$ is compact on BMOA by Corollary 5.2, and thus $C_{\phi}=C_{\psi^{-1} \circ \phi} C_{\psi}$ is compact as well.

Madigan and Matheson raise the following question in [7].

Is there a $\phi$ in the little Bloch space such that $\overline{\phi(U)} \cap \partial U$ is infinite and

$C_{\phi}$ is compact on the little Bloch space?

Corollary 5.2 together with Proposition 6.1 provide an affirmative answer to this question. In fact, one can construct a mapping $\phi$ such that $C_{\phi}$ is compact on the little Bloch space and $\overline{\phi(U)}=\bar{U}$. Let $\left(t_{n}\right)$ be a sequence of real numbers such that $\left\{e^{i t_{n}}: n=1,2, \ldots\right\}$ is dense in $\partial U$, and let $\left(h_{n}\right)$ be a sequence of positive numbers less than, say, $1 / 2$ such that $\sum_{k=1}^{\infty} h_{k}<\infty$. For each positive integer $k$, 
let $E_{k}$ denote the open region in the right-half disk bounded between the curve $y=h_{k}(x-1)^{2}$ and its reflection in the $x$-axis. Set

$$
G=(1 / 2) U \cup\left(\bigcup_{k=1}^{\infty} e^{i t_{k}}\left(E_{k}\right)\right) .
$$

Observe that $G$ is star-like with respect to the origin, that $\bar{G}=\bar{U}$, and that $G$ makes mean-order contact $1 / 2$ with $\partial U$. Thus, by Corollary 5.2, a Riemann mapping $\phi$ of $U$ onto $G$ will induce a compact composition operator on BMOA. Moreover, because $\phi$ is univalent it will be in VMOA and hence will induce a compact composition operator on VMOA. Finally, compactness of $C_{\phi}$ on VMOA implies its compactness on the little Bloch space (see Proposition 6.1 of the following section).

\section{FURTHER COMPACTNESS COMPARISONS}

Recall that the Bloch space $\mathcal{B}$ consists of those analytic functions on $U$ for which

$$
\|f\|_{\mathcal{B}}=\sup _{z \in U}\left(1-|z|^{2}\right)\left|f^{\prime}(z)\right|
$$

is finite and the little Bloch space $\mathcal{B}_{0}$ is that subspace of $\mathcal{B}$ comprising functions $f$ for which

$$
\lim _{|z| \rightarrow 1}\left(1-|z|^{2}\right)\left|f^{\prime}(z)\right|=0 .
$$

It is well known that BMOA $\subset \mathcal{B}$ and that $\mathrm{VMOA} \subset \mathcal{B}_{0}$ (see, e.g. [8, p. 93]).

Madigan and Matheson [7] characterize compactness of $C_{\phi}$ on $\mathcal{B}$ and $\mathcal{B}_{0}$ as follows. The operator $C_{\phi}$ is compact on $\mathcal{B}_{0}$ if and only if

$$
\lim _{|z| \rightarrow 1} \frac{1-|z|^{2}}{1-|\phi(z)|^{2}}\left|\phi^{\prime}(z)\right|=0
$$

The operator $C_{\phi}$ is compact on $\mathcal{B}$ if and only if for each $\epsilon>0$ there exists an $r$, $0<r<1$, such that

$$
\frac{1-|z|^{2}}{1-|\phi(z)|^{2}}\left|\phi^{\prime}(z)\right|<\epsilon
$$

whenever $|\phi(z)|>r$.

Observe that there is a similarity in form between these Bloch space compactness results and our compactness results for BMOA and VMOA.

M. Tjani recently proved [14, Theorem 3.1] that $C_{\phi}$ is compact on $\mathcal{B}$ if and only if

$$
\lim _{|\lambda| \rightarrow 1}\left\|C_{\phi} \alpha_{\lambda}\right\|_{\mathcal{B}}=0
$$

where $\alpha_{\lambda}$ is the disk automorphism taking $z$ to $\frac{\lambda-z}{1-\lambda z}$. Using this characterization of compactness for Bloch-space composition operators, Tjani shows

- if $C_{\phi}$ is compact on BMOA, then $C_{\phi}$ is compact on the Bloch space.

The authors of the present paper have obtained an alternative proof of Tjani's result, showing that if our BMOA compactness condition holds, then Madigan and Matheson's $\mathcal{B}$ compactness condition holds (we omit the proof).

Wayne Smith [12] recently constructed an inner function $\phi$ such that $C_{\phi}$ is compact on the little Bloch space. Since no inner function can induce a compact compo- 
sition operator on BMOA (because, e.g., BMOA compactness implies Hardy-space compactness), Smith's example shows that compactness of $C_{\phi}$ on the little Bloch space and a fortiori on the Bloch space doesn't imply its compactness on BMOA.

Our contribution to the Bloch-BMOA compactness comparison is the following.

Proposition 6.1. Suppose that $C_{\phi}$ is compact on VMOA. Then

$$
\lim _{|a| \rightarrow 1^{-}} \frac{\left|\phi^{\prime}(a)\right|(1-|a|)}{1-|\phi(a)|}=0 .
$$

so that $C_{\phi}$ is compact on the little Bloch space.

We present a simple, direct proof. Let $E_{a}^{\prime} \in \mathrm{VMOA}^{*}$ be defined by $E_{a}^{\prime}(f)=$ $f^{\prime}(a)$. For $|\zeta|=1$ and $r \in[0,1)$, let

$$
L_{r \zeta}(z)=\log (1-r \zeta z) .
$$

Lemma 6.2. There are positive constants $C_{1}$ and $C_{2}$ such that

$$
\frac{C_{1}}{1-|a|} \leq\left\|E_{a}^{\prime}\right\| \leq \frac{C_{2}}{1-|a|} .
$$

Proof. The inequality on the right follows from the containment of VMOA in the Bloch space. That on the left follows from the finiteness of

$$
\sup \left\{\left\|L_{r \zeta}\right\|_{*}:|\zeta|=1 \text { and } 0 \leq r<1\right\}
$$

and that the observation that $\left|E_{a}^{\prime}\left(L_{r \bar{a} /|a|}\right)\right|=r /(1-r|a|)$.

Proof of Proposition 6.1. By the preceding lemma, the set $\left\{(1-|a|) E_{a}^{\prime}: a \in U\right\}$ is bounded in VMOA* . Moreover, for each $f$ in VMOA, $\lim _{|a| \rightarrow 1}(1-|a|) E_{a}^{\prime}(f)=0$. Hence, because $C_{\phi}^{*}$ is compact, we must have

$$
\begin{aligned}
0 & =\lim _{|a| \rightarrow 1}\left\|(1-|a|) C_{\phi}^{*}\left(E_{a}^{\prime}\right)\right\| \\
& =\lim _{|a| \rightarrow 1}(1-|a|)\left|\phi^{\prime}(a)\right|\left\|E_{\phi(a)}^{\prime}\right\|
\end{aligned}
$$

Because $\left\|E_{\phi(a)}^{\prime}\right\| \geq \frac{C_{1}}{(1-|\phi(a)|)}$, Proposition 6.1 follows.

We remark that our argument yielding Proposition 6.1 also provides an alternative proof that

$$
\lim _{|a| \rightarrow 1^{-}} \frac{\left|\phi^{\prime}(a)\right|(1-|a|)}{1-|\phi(a)|}=0
$$

is necessary for the compactness of $C_{\phi}$ on the little Bloch space.

We conclude with a question. Madigan and Matheson show [7, Theorem 3] that weak compactness of $C_{\phi}$ on $\mathcal{B}_{0}$ implies its compactness on $\mathcal{B}_{0}$.

Suppose that $C_{\phi}:$ VMOA $\rightarrow$ VMOA is weakly compact; i.e., suppose that $C_{\phi}$ maps BMOA into VMOA. Must $C_{\phi}: \mathrm{VMOA} \rightarrow$ VMOA be compact?

To contrast our question with its analogue for the little Bloch space, we offer the following remarks. That weak compactness implies compactness for composition operators on $\mathcal{B}_{0}$ follows from the more general result that any weakly compact operator $T: \mathcal{B}_{0} \rightarrow X$ is compact, where $X$ is any Banach space. This general result has a simple functional analytic proof based on the fact that the dual of $\mathcal{B}_{0}$ is isomorphic to the the Bergman space $L_{a}^{1}(U)$ (by [5, Theorem 10]; see also 
Theorem 2.4 (b) of [1]). Because $L_{a}^{1}(U)$ has the Schur property (every weakly convergent sequence in $L_{a}^{1}(U)$ is norm convergent [4, Theorem 12, p. 295]), every weakly compact operator with range in $L_{a}^{1}(U)$ must be compact. Since an operator is compact (or weakly compact) if and only if its adjoint is, the result follows. On the other hand, no such argument is possible for VMOA, as its dual space, which is isomorphic to $H^{1}$, contains complemented copies of Hilbert space (cf. [15, p. 21]).

\section{ACKNOWLEDGMENT}

The authors would like to thank the referee for providing a number of helpful comments and corrections.

\section{REFERENCES}

[1] J. M. Anderson, J. Clunie, and Ch. Pommerenke, On Bloch functions and normal functions, J. Reine Angew. Math. 270 (1974), 12-37. MR 50:13536

[2] J. Cima and A. Matheson, Essential norms of composition operators and Alexandrov measures, Pacific J. Math., 179 (1997), 59-64. MR 98e:47047

[3] C. C. Cowen and B. D. MacCluer, Composition Operators on Spaces of Analytic Functions, CRC Press, Boca Raton, 1995. MR 97i:47056

[4] N. Dunford and J. Schwartz, Linear Operators, John Wiley and Sons, New York, 1988.

[5] P. L. Duren, B. W. Romberg, and A. L. Shields, Linear functionals on $H^{p}$ spaces with $0<p<1$, J. Reine Angew. Math. 238 (1969). 32-60. MR 41:4217

[6] J. B. Garnett, Bounded Analytic Functions, Academic Press, New York, 1981. MR 83g:30037

[7] K. Madigan and A. Matheson, Compact composition operators on the Bloch space, Trans. Amer. Math. Soc. 347 (7), 1995, 2679-2687. MR 95i:47061

[8] D. Sarason, Function Theory on the Unit Circle, Lecture notes, Virginia Polytechnic and State University, 1978. MR 80d:30035

[9] J. H. Shapiro, The essential norm of a composition operator, Annals of Math. 125 (1987), 375-404. MR 88c: 47058

[10] J. H. Shapiro, Composition Operators and Classical Function Theory, Springer-Verlag, New York, 1993. MR 94k:47049

[11] J. H. Shapiro and P. D. Taylor, Compact, nuclear, and Hilbert Schmidt composition operators on $H^{2}$, Indiana Univ. Math. J. 23 (1973), 471-496. MR 48:4816

[12] W. Smith, Inner functions in the hyperbolic little Bloch class, Michigan Math. J. 45 (1988), 103-104. CMP 98:11

[13] K. Stephenson, Weak subordination and stable classes of meromorphic functions, Trans. Amer. Math. Soc. 262 (1980), 565-577. MR 81m:30029

[14] M. Tjani, Compact composition operators on some Möbius invariant Banach spaces, Thesis, Michigan State University, 1996.

[15] P. Wojtaszczyk, Banach Spaces for Analysts, Cambridge University Press, Cambridge, 1991. MR 93d:46001

Department of Mathematics, Washington and Lee University, Lexington, Virginia 24450

E-mail address: pbourdon@wlu.edu

Department of Mathematics, University of North Carolina, Chapel Hill, North CarOLINA 27599

E-mail address: cima@math.unc.edu

Department of Mathematics, Lamar University, Beaumont, Texas 77710

E-mail address: matheson@math.lamar.edu 